\title{
Separation Anxiety in Canines and Potential Mitigation Options
}

\author{
Danielle Rose Schlehahn*
}

\begin{abstract}
Separation anxiety is a very common disorder that many domestic dogs suffer from. It comes second only to aggression in terms of behavioural disorders reported and is the most common anxiety disorder in dogs. Canine separation anxiety has a vast range of symptoms, including excessive vocalisation, destruction, inappropriate elimination, hypersalivation, and restlessness. Since these symptoms only occur in the absence of the owner and vary between dogs, separation anxiety is exceedingly difficult to diagnose. This disorder is potentially affected by numerous factors, including gender, breed, existing behavioural issues, the degree of attachment to the owner, and environmental factors, such as the source of the dog. Fortunately, options for anxiety mitigation exist, including proper training of the dog, as well as medications such as fluoxetine and clomipramine. It is imperative that a proper diagnosis and treatment is given to the dog early in the condition, as this disorder can negatively affect both the mental and physical health of the dog, as well as the owner-dog relationship. Unfortunately, the daily struggles of managing dogs with such a disorder take a toll on the owners. This potentially results in the all too common decision to surrender the dog to a shelter, and potentially euthanasia. Ultimately, this disorder still requires future research in many aspects, in order to better the lives of both the dog and its owners.
\end{abstract}

Keywords: separation anxiety, anxiety mitigation, canine, behavioural problems

\subsection{Introduction}

Behavioural issues are exceedingly common in today's domestic dog population (Martinez et al. 2011). Dogs often form such a strong bond with their owners that they will do anything in their power to prevent being separated from them. Their reactions could be as minute as vocalizing their frustrations of being left behind, or, more seriously, chewing on door knobs or jumping through windows in an attempt to reunite with their owners (Sherman and Mills 2008). This condition is called separation anxiety. The purpose of this thesis is to explore the factors that are associated with canine separation anxiety and potential mitigations that could assist in the rehabilitation of these dogs.
Like most other behavioural problems, canine separation anxiety can affect the welfare and quality of life of both the dog and its owner (Sherman and Mills 2008). Separation anxiety is the most common form of anxietyrelated condition in canines and comes second only to aggression in reported behavioural conditions in dogs (Bamberger and Houpt 2006). It is estimated that this disorder is prevalent in between 20 and 40 percent of all dogs that are treated by animal behaviour specialists in North America (Simpson 2000, as reviewed by Thielke and Udell 2017). Dogs with separation anxiety exhibit unwanted

\footnotetext{
*Department of Animal Science, College of Agriculture and Bioresources, University of Saskatchewan, Saskatoon, SK, Canada Correspondence: drs578@mail.usask.ca
} 


\begin{tabular}{|c|c|c|c|}
\hline \multicolumn{4}{|c|}{ Dogs with separation anxiety } \\
\hline Symptom & $\begin{array}{l}\text { Amount of } \\
\text { dogs }\end{array}$ & $\begin{array}{l}\text { Moderate to } \\
\text { severe (\%) }\end{array}$ & Owner unsure (\%) \\
\hline Destruction & 198 & 71.7 & 1.0 \\
\hline Vocalization & 178 & 61.2 & 11.0 \\
\hline Elimination & 192 & 28.1 & 4.0 \\
\hline
\end{tabular}

Table 2.1: Symptoms noted by owners of dogs with separation anxiety, compared to control dogs, during and around departure and arrival of owner (Based on information from Flannigan and Dodman 2001).

behaviours only when the owner is away (Blackwell et al. 2016), and often suffer from serious separation anxiety panic, showing characteristics consistent with the condition after being left alone for only minutes (Overall et al. 2001).

Simpson et al. (2007) stated that separation anxiety in canines results when the dog is separated from its owners or other familiar people. The most common behaviours associated with separation anxiety are excessive vocalization and destructiveness (Simpson et al. 2007). The latter behaviour typically involves dogs scratching or chewing furniture, and this enactment is often located near the area where the owner was last seen (Blackwell et al. 2006). In addition to these symptoms, dogs may also rearrange household objects or exhibit hypersalivation, selfmutilation, repetitive behaviour, inappropriate urination and defecation, or restlessness (Blackwell et al. 2006; Sherman 2008). Clearly, the symptoms of separation anxiety are nonspecific. This results in a challenge when attempting to diagnose this behavioural problem (Overall et al. 2001).

Fortunately, there are many mitigations proposed to assist dogs with separation anxiety. These include training the dog or instructing the owner on how to properly manage the condition with a variety of techniques (Voith and Borchelt 1996; Takeuchi et al. 2000; Blackwell et al. 2006; Blackwell et al. 2016). Medications can also be administered, such as clomipramine (King et al. 2004; Gaultier et al. 2005; Cannas et al. 2014) or fluoxetine (Simpson et al. 2007; Karagiannis et al. 2015). Treating separation anxiety will benefit both the dog and the relationship with its owner, as well as minimize the number of dogs surrendered to shelters (Thielke and Udell 2017). Ultimately, separation anxiety is an extensive disorder that needs sufficient treatment for the sake of both the dog and its owner.

\subsection{Diagnosing Separation Anxiety}

Due to the multitude and variety of symptoms of separation anxiety, it is often difficult to form a correct diagnosis. Flannigan and Dodman (2001) investigated 200 dogs diagnosed with separation anxiety and outlined the common behavioural problems that these dogs exhibited when they were left home alone. Dog owners were asked to fill out an eight-page behavioural history form before the dogs were clinically evaluated and a diagnosis determined. The study results displayed in Table 2.1 show that the most common problem in dogs with separation anxiety was destruction of the home (71.7\%), followed by excessive vocalization (61.2\%), and inappropriate elimination (28.1\%). It should be noted that these were observations reported by the owners, and many behaviours, like signs of depression or excessive vocalization, would be difficult or impossible to note while the owners are absent. Although not mentioned in this study, perhaps neighbours notified the owner of excessive barking; regardless, accuracy cannot be assumed unless further testing is performed and a more verifiable observation method is utilized, such as videotaping.

Sherman (2008) described similar findings of the most common symptoms of separation anxiety. Figure 2.1 shows destruction by a dog with separation anxiety near the exit point of its owner. Figure 2.2 depicts the physical damage that dogs can inflict on themselves (Sherman 2008).

More recently, Storengen et al. (2014) performed a descriptive study involving 215 dogs that were diagnosed with separation anxiety in a behaviour clinic in Norway. Clinical observations, owner interviews, and questionnaires were used to draw conclusions. The study examined the most common behaviours depicted by the dogs when left home alone, which can be seen in Table 2.3. The authors found similar results to Flannigan and Dodman (2001), in that vocalization was the most common complaint, followed by destruction and excessive motor activity. Storengen et al. (2014) reported elimination as separate entities of urine and stool, so excessive motor activity surpassed this as the thirdmost-reported symptom. Similar to the study by Flannigan and Dodman (2001), vocalization and motor activity were difficult to observe without video evidence during the owner's absence. Storengen et al.'s (2014) study was enhanced in that it also utilized results from the behaviour clinic. Similar results were found in Bradshaw et al.'s (2002) longitudinal study of litters containing 23 Labrador 


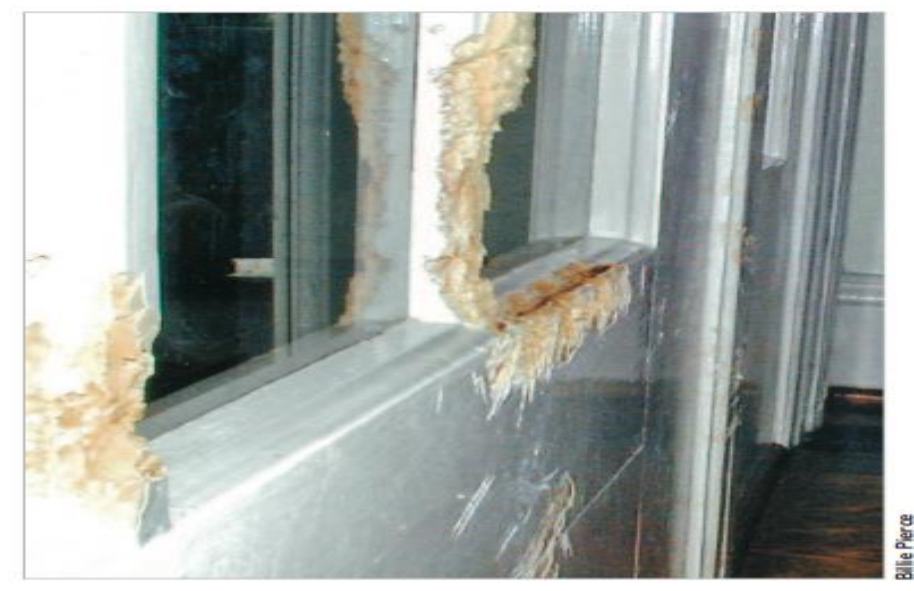

Figure 2.1: Evidence of a dog with separation anxiety destroying an area near the door through which the owner exited (Sherman 2008)

Retrievers and 17 Border Collies. The breeder and new owners answered questions during interviews at different stages of the puppy's lives, including how the dog and owner interacted and separation score.

This study also collected data randomly for a crosssectional study from 105 dog owners that were walking their dogs. The cross-sectional study found that barking was the

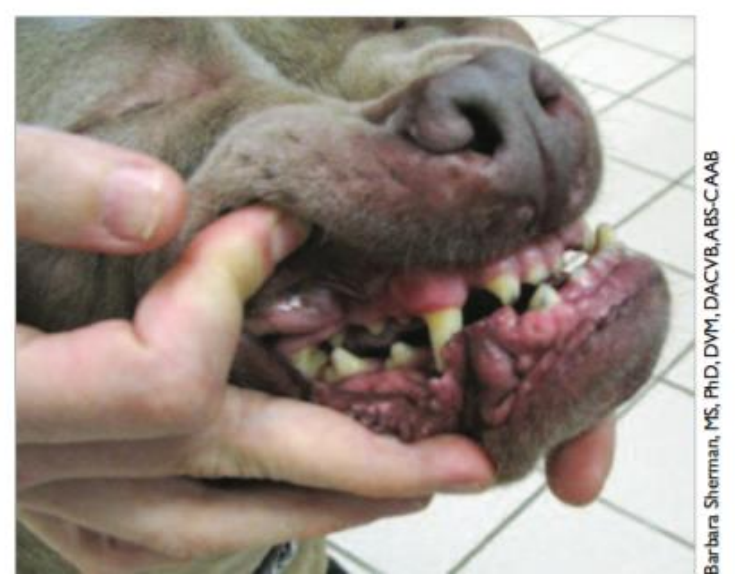

Figure 2.2: Damage done to the teeth of a dog with separation anxiety after it tried to bite through a crate that it was confined to. The dog had to be crated because it previously leaped through a second-story screen trying to escape when left alone (Sherman 2008)

most common behaviour (15\%), with destruction (11\%) trailing closely behind. Judging from the similarity between Flannigan and Dodman (2001), Storengen et al. (2014), Bradshaw et al. (2002), and others (Overall et al. 2001; Palestrini et al. 2010), destruction, vocalization, elimination, and excessive motor activity are frequent symptoms of canine separation anxiety and could lead to a diagnosis of this disorder.

\begin{tabular}{|l|l|l|}
\hline \multicolumn{2}{|l|}{ Distribution of behaviours in the SA-dogs } \\
\hline Behaviour & Amount of dogs & Relative distribution (\%) \\
\hline Vocalization & 163 & 83.2 \\
\hline Destruction & 71 & 36.4 \\
\hline Excessive motor activity & 51 & 26.2 \\
\hline Elimination of urine & 37 & 18.7 \\
\hline Elimination of stool & 23 & 11.7 \\
\hline Hypersalivation & 12 & 6.1 \\
\hline Vomiting & 2 & 1 \\
\hline Self-trauma & 1 & 0.5 \\
\hline
\end{tabular}

Table 2.3: The most common separation anxiety related behaviours when dogs were left home alone and their relative distributions $(n=198)$ (Based on information from Storengen et al. 2014) 


\subsection{Diagnostic Model}

Despite these symptoms being noticeably displayed by a dog, they do not allow for a clear diagnosis, as they can also be indicative of other behavioural problems, such as territorialism or obsessive-compulsive disorder. Attempting to standardize a diagnosis based on specific parameters, Appleby and Pluijmakers (2004) proposed a model that divides types of separation anxiety into three classes: $A, B$, or $C$. These classes are important because the appropriate treatment regimen is dictated by the class of separation anxiety that a dog falls in. Class $A$ includes dogs that have shown primary hyperattachment to their owner from puppyhood. These dogs follow their owners around the house and require constant physical contact when their owners are around. They can predict their owner's departure through certain cues like picking up their keys and show distress in anticipation of separation. When the owner is away, class A dogs show destructive behaviours involving the owner's exit point and items with a strong owner scent. They also exhibit excessive vocalization. This group requires treatment in reducing the dog's dependence on the owner and increasing their independence to regain emotional homeostasis. This can be done by transferring the dependence on the owner to another stimulus, such as a toy. This treatment should be introduced gradually in phases (Appleby and Pluijmakers 2004).

Class B dogs exhibit secondary hyperattachment and are normal as puppies. These dogs depend on one or more social stimuli, such as the owner, or non-social stimuli, like an area in the house. When their owner is preparing to leave, they exhibit distress. If the dog is over-dependent on the owner, rather than an environmental stimulus, then the anxiety the dog experiences when the owner leaves increases the possibility for fear. Class B dogs may try to prevent the owner from leaving and become depressed in anticipation of punishment due to destructive behaviour when the owner returns. When the owner is gone, the dog may try to escape or destroy the exit point of the owner. They also exhibit inappropriate elimination and vocalization. Treatment for this group includes identifying and, if possible, eliminating fearful stimuli such as prolonged departure of the owner. Reducing the reliance on the owner and potentially transferring this reliance to another stimulus like toys will also alleviate the disorder (Appleby and Pluijmakers 2004).

Finally, class $C$ dogs develop separation anxiety at any age in response to a fearful event; for example, a thunderstorm can be a trigger for some dogs. No inappropriate dependency behaviours are seen and they react to the fearful stimulus even in the presence of the owner. The reaction may be diminished when the owner is present, however. The dogs develop stress signs by being able to predict the owner's departure and associate it with the fearful stimulus. Class C dogs will inappropriately eliminate while owner is gone, and exhibit other symptoms such as destruction, trying to cope, escape, or hide (Appleby and Pluijmakers 2004). Treatment of class $C$ dogs involves helping an animal cope with the underlying fear, rather than its attachment with the owner. This includes systematic desensitization and counterconditioning the dog's response to the stimulus that is eliciting the fear response (Simpson 2000, as reported by Appleby and Pluijmakers 2004). These treatments, as well as others, will be discussed in the coming sections.

This model can be extremely helpful, in that it bases a diagnosis on more than just the presence of symptoms. It goes further in that it categorizes the symptoms into different treatable groups and lists the timeline and possible triggers of the symptoms. These details are important in determining possible mitigations for separation anxiety.

\subsection{Factors Affecting Separation Anxiety}

Canine separation anxiety is known to be associated with elements other than biological factors. Horwitz and Mills (2009) explained that the causes of separation anxiety are multifactorial, meaning that it is affected by both the environment and genetics. They stated that an inherited predisposition of the disorder may be due to humans selectively breeding dogs for social connection and dependence on humans. The underlying causations include fear, anxiety, over-attachment, or a lack of stimulation. Numerous other factors have been reported, including a history of being left alone for long periods of time, long periods without being left alone, or kennel housing (Voith and Borchelt 1996).

Separation anxiety can also be induced by seemingly trivial, everyday actions of a dog's owner. Similar to what Appleby and Pluijmakers (2004) explained, Amat et al. (2014) stated that dogs can learn to predict their owner's departure, which may add to the anxiety response. Dogs learn to associate when humans are departing and several clues that the owners give prior to leaving. These clues are often performed unconsciously by the owner, such as picking up house keys or putting on a coat. The advice commonly given to owners in the past has been to discourage this predictability (Takeuchi et al. 2000). However, conflicting evidence is presented by Amat et al. (2014), who postulated that predictability actually reduces the anxiety associated with separation. This may be because the dog knows that it is possible to relax in the absence of the owner's departure cues, so it believes that it has control over its environment. 
Then, when the departure cues are observed, the dog knows that the owner is departing and can prepare itself (Amat et al. 2014). However, this was hypothesized based on human cases of separation anxiety and should be further tested for its applicability to canines. In a case-control study, Flannigan and Dodman (2001) agreed with Amat et al. (2014). They found that dogs with separation anxiety were four and a half to five times more likely to exhibit anxiety while their owners were preparing to depart, which indicates hyperattachment. However, the authors did not discuss whether this owner predictability should be encouraged or discouraged.

\subsection{Attachment Differences}

Dogs can vary in how strong their attachment is to their owner. This can be observed by noting how the dogs act around their owners. The strength of attachment level influences the severity of their separation anxiety and can help to categorize the dog in either class A, B, or C, according to the model by Appleby and Pluijmakers (2004). It is important to determine the source of this attachment and how it influences separation anxiety. Thielke and Udell (2017) note that oxytocin is a hormone involved in forming an attachment between humans and dogs. This attachment can vary in strength. However, it can be difficult to determine what type of attachment style is depicted by the dogs. Parthasarathy and Crowell-Davis (2006) determined that hyperattachment presents as an overdependence of the dog on the owner, and the dog showing a desperate need to keep close proximity to them, even after they have departed. In this case, the dog tends to keep close to the door that the owner exited through. Flannigan and Dodman (2001) speculated that hyperattachment may also be indicated by how intense the dog's anxiety response to cues while their owners are departing. Additionally, they also explained that dogs who suffer from hyperattachment will also excessively greet their owners upon their arrival. A few studies have attempted to determine if hyperattachment can be a useful diagnostic of separation anxiety.

Flannigan and Dodman (2001) performed a casecontrol study that examined the correlation between hyperattachment and canine separation anxiety. They determined that dogs diagnosed with separation anxiety were three times more likely to follow their owners around incessantly upon arrival and almost four times more likely to greet them excitedly for two or more minutes, compared to dogs without the disorder. These results support the principle that hyperattachment is indeed a significant component of separation anxiety and should be used as a diagnostic tool. However, these results may not be representative of the entire dog population. This study looked at 200 dogs diagnosed with separation anxiety and
200 control dogs. The control cases were sampled from dogs in other behavioural-related cases (Flannigan and Dodman 2001). This detail could have potentially altered the results because the sample is only coming from owners already concerned about behavioural problems. These behavioural problems could include any number of disorders, some of which would mean that a dog in the control group already avoids its owner. For example, fearfulness may result in a dog actively avoiding its owner. Future studies should be mindful of using an appropriate source of dogs.

Results from Parthasarathy and Crowell-Davis (2006) differed from those obtained above. In this study, the owner and dog attachment style of 75 dogs was investigated. The dogs had been obtained by the owners at no more than four months of age and were between two and eight years old during the period of the trial. This ensured that the dogs had been with the owners for enough time to potentially build an attachment. The dogs were prescreened to prevent the inclusion of subjects who were under treatment for other behavioural problems, unlike the previous study's protocol. Thirty-one dogs had clinical separation anxiety reported by their owners in an Anxiety Survey, and forty-four did not. There was no significant difference $(P>0.05)$ between the duration of contact with owners and the proximity to owners after they were reunited displayed by dogs with separation anxiety compared to dogs without. This lack of significance is indicative that maintenance of proximity is not an essential parameter of separation anxiety in canines and should not be used as a diagnostic tool during a clinical assessment. It was found that 65 percent of the dogs without separation anxiety still followed their owners infallibly. Interestingly, the data also showed that a separation anxiety diagnosis and hyperattachment were not correlated in any way. Moreover, no difference was seen in the dogs maintaining close proximity to the door that their owner departed through when the group with separation anxiety was compared to the control group, meaning this is also not a reasonable diagnostic of separation anxiety (Parthasarathy and CrowellDavis 2006). Animals chosen for this study are considered to comprise a more representative sample than those of Flannigan and Dodman (2001) for a number of reasons. First, they recruited the owners through fliers and word of mouth without revealing the variables. This resulted in a nonbiased sample group and more representative data. Additionally, they prescreened for other behavioural issues so that this would not alter the results, whereas Flannigan and Dodman (2001) obtained their control dogs out of existing behavioural cases. Clearly, making an accurate diagnosis of this behavioural disorder is difficult and is influenced by many factors which must be accounted for to yield accurate results. 


\subsection{Gender}

The gender of the canine could be a contributing factor in a separation anxiety diagnosis. For example, in a cohort study, Takeuchi et al. (2000) found that there were significantly more males than females that showed signs of separation anxiety when they investigated the treatment outcome in 52 dogs diagnosed with the disorder. Bradshaw et al. (2002), Bamberger and Houpt (2006), McGreevy and Masters (2007), and Storengen et al. (2014) all provided data in agreement with Takeuchi et al.'s (2000) study.

Despite many studies stating that gender affects separation anxiety, others disagree. In a 2001 case study, Overall et al. (2001) investigated 141 dogs diagnosed with separation anxiety, noise phobia, or thunderstorm phobia. Among other things, they concluded that separation anxiety was not affected by the gender of the dog. Furthermore, Flannigan and Dodman (2001) found that, when comparing 200 dogs diagnosed with separation anxiety to 200 that did not have it, 58 percent of the control dogs and 60 percent of the dogs with separation anxiety were male. This would suggest that it is more common for a male to have separation anxiety, as noted by many other studies (Takeuchi et al. 2000; Bradshaw et al. 2002; Bamberger and Houpt 2006; McGreevy and Masters 2007; Storengen et al. 2014). However, Flannigan and Dodman (2001) noted that gender was not found to be a significant factor that affects separation anxiety. Regardless of their findings, the authors highlighted that many other studies have found that males represent roughly 60 percent of the dogs with behavioural issues like separation anxiety. This could be because males are more prone to behavioural problems or that they are more severely affected, resulting in their owners seeking treatment more often. These varying results would suggest that more testing is needed before a confirmed conclusion regarding gender can be drawn.

\subsection{Breed}

Like gender, the breed of the canine in question has affected reported separation anxiety cases in some studies. Bamberger and Houpt (2006) tested 1,644 dogs with various behavioural problems and diagnosed 236 with separation anxiety. The authors looked at signalment factors, such as breed and gender, that may affect separation anxiety. It was found that Beagles, Dalmatians, and mixed-breed dogs were diagnosed with separation anxiety more often than expected. The number of mixed-breed dogs (104) exhibiting separation anxiety was much larger than the group of purebred dogs (17) that had separation anxiety. The paper does not present these results on a percentage basis, however. It should also be noted that the dogs in this study were obtained from patients of veterinary behaviorists and that the breed distributions may not accurately depict actual breed occurrence of separation anxiety (Bamberger and Houpt 2006). Takeuchi et al. (2001) found similar results when they compared breeds with separation anxiety to the total dog population of the teaching hospital that performed the experiment. It was found that there was a significantly larger proportion of mixed breed dogs $(48.7 \%)(P<0.002)$ in the group of dogs with separation anxiety compared to the total hospital population (23\%). Of the purebreds, the most commonly represented breeds included Labrador Retriever, Dalmatian, Cocker Spaniel, English Springer Spaniel, and Beagle.

More recently, Sherman and Mills (2008) also found that the prevalence of separation anxiety was not higher in any specific breed, apart from mixed-breed dogs, which Storengen et al. (2014) agreed with. Despite mixed-breeds being the most represented, certain purebreds were commonly diagnosed with separation anxiety as well. Table 3.1 illustrates the top 10 breeds diagnosed with separation anxiety in Norway and indicates that, aside from mixedbreed dogs, the top three reported breeds were the Cocker Spaniel, Gordon Setter, and Schnauzer. Storengen et al. (2014) suggested that the difference in breed prevalence may be due to a buildup of risk genes and alleles in certain breeds.

Similarly, Flannigan and Dodman (2001) found that more mixed-breed dogs had separation anxiety than purebreds. However, this finding showed no significant differences in level of separation anxiety existed between the two dog types. Of the purebreds, the highest reported breeds in the separation anxiety group, in order of subsequent incidence, were German Shepherds, Labrador Retrievers, Golden Retrievers, English Springer Spaniels, and English Cocker Spaniels.

Aside from the agreement that mixed breeds suffer from separation anxiety more than purebred breeds, it is evident that the results above do not coincide with each other in regard to which pure breed is most represented overall. This could be due to a few factors. Firstly, canine breed popularity evolves rapidly depending on public demand. Thus, certain breeds represent a higher portion of the population, depending on the year that studies are performed. This could mean that a study would see a higher incidence of separation anxiety in a certain breed when they are just owned in larger quantities by the general population than others. Storengen et al. (2014) highlighted this point by comparing the percentages of the top ten 


\begin{tabular}{|l|l|l|l|}
\hline Small animal clinic & NKC: $2005-2010$ & Behaviour clinic (SA) & Behaviour clinic (all patients) \\
\hline Mixed breed & German shepherd & Mixed breed & Mixed breed \\
\hline Labrador Retriever & Norwegian elkhound & Cocker spaniel & German shepherd \\
\hline German shepherd & Golden retriever & Gordon setter & Jack Russell terrier \\
\hline English setter & $\begin{array}{l}\text { Cavalier King Charles } \\
\text { spaniel }\end{array}$ & Schnauzer & Border collie \\
\hline Rottweiler & Border collie & Dachshund, long-haired & Golden retriever \\
\hline Boxer & English setter & Jack Russell terrier & Rottweiler \\
\hline Jack Russell terrier & Gordon setter & Dachshund, short-haired & English springer spaniel \\
\hline Standard poodle & Labrador retriever & German shepherd & Flat-coated spaniel \\
\hline Flat-coated retriever & Tibetan spaniel & Tibetan spaniel & Dachshund, long-haired \\
\hline $\begin{array}{l}\text { Cavalier King Charles } \\
\text { spaniel }\end{array}$ & Irish setter & Rhodesian ridgeback & \\
\hline
\end{tabular}

Table 3.1: The top ten breeds diagnosed with separation anxiety at a behaviour clinic based on percentages, compared to the top ten most common breeds at a behaviour clinic, registered in the Norwegian Kennel Club (NKC), and patients at a small animal clinic (Based on information from Storengen et al. 2014)

breeds diagnosed with separation anxiety to other parameters, including the top ten most popular breeds that visited a behaviour clinic, registered in the Norwegian Kennel Club (NKC) at the time, and visited a small animal clinic (Table 3.1). In this particular study, Cocker Spaniels were most frequently diagnosed with separation anxiety, despite this breed only representing 1.8 percent of the dogs registered in the NKC and not being in the top ten breeds registered in the NKC. Additionally, Schnauzers represented 2.8 percent of the total dogs at the behaviour clinic while only making up 0.3 percent of the dogs registered in the NKC. This means that this breed could truly be more frequently affected than others.

Another factor that could have skewed these findings is the source of the dog. Sherman and Mills (2008) explained that the source of the dog needs to be factored into determining if breed really does correlate with a diagnosis. In many studies, there are more dogs diagnosed with separation anxiety that come from shelters, and mixed-breed dogs are more likely to be found in shelters (Sherman and Mills 2008). Shelter environments and the impact on separation anxiety will be discussed in more detail below.

\subsection{Other Behavioural Issues}

Other existing behavioural conditions have been found to correlate with canine separation anxiety (Horwitz and Mills 2009). For example, McGreevy and Masters (2007) released a questionnaire asking owners to rank their dog's misbehaviours relating to separation-related distress on a scale of zero to five. Six hundred and ninety dogs were used in the analysis. The data showed that dogs which suffered from excessive excitability had a greater chance of having a higher separation anxiety score $(P=0.008)$. Similarly, dogs with high fear levels had an increased probability of having a high separation anxiety score $(P=0.001)$. This study was successful in that they used a very large sample size, ensuring that the results are representative. In addition to these behaviours, Storengen et al. (2014) found that compulsive behaviours and coprophagia, or consuming their own feces, were common in dogs diagnosed with separation anxiety.

3.4.1 Noise and Thunderstorm Phobia. Canine separation anxiety is commonly associated with noise and thunderstorm phobia. Overall et al. (2001) asked 51 dog owners about separation anxiety symptoms in their dogs, as well as whether the dogs reacted to thunderstorms, fireworks, or other general noises. They found that 21 dogs had both separation anxiety and noise or thunderstorm phobia, and that dogs with multiple conditions had more severe symptom. The authors reported that dogs which always reacted fearfully to noises were more likely to exhibit behaviours associated with separation anxiety, such as destruction, vocalization, or urination, than dogs which reacted to noises less often. In a second part of the study, the authors used records of 141 dogs known to have separation anxiety, noise phobia, or thunderstorm phobia. The records showed that 41 dogs had only separation anxiety, 1 had thunderstorm phobia, 1 had noise phobia, 7 had both separation anxiety and thunderstorm phobia, 21 had separation anxiety and noise phobia, 10 had thunderstorm and noise phobia, and 60 had all 3 conditions. The authors stated that the frequency of dogs having all three conditions was significantly higher than would be expected if they were truly independent. Dogs with all three conditions suffered from more intense signs of distress than those with only one. In this second part of the study, the authors determined that the probability that a dog already diagnosed with noise phobia would also have separation anxiety was determined to be high (0.88) and almost equivalent to the probability that a dog already having thunderstorm phobia would also have separation anxiety (o.86). This suggests that both noise and thunderstorm 
phobia have strong potential to predispose a dog to separation anxiety. Interestingly, the probability of a dog diagnosed with separation anxiety also having noise phobia (0.63) was higher than the probability of a dog with separation anxiety also having thunderstorm phobia (0.52).

Based on these results, the probability of noise phobia occurrence in conjunction with separation anxiety was much higher than expected if these were two independent conditions. These correlations support the author's hypothesis that dogs showing unfavorable reactivity to noises in general have the potential to be predisposed to separation anxiety.

Comparably, Storengen et al. (2014) sought out other reported behavioural problems in 215 dogs diagnosed with separation anxiety. They found that fear of noises was the largest group of observed behaviours in dogs with separation anxiety. It was discovered that 94 (43.7\%) of these dogs had a fear of noises. Similar results were found in Flannigan and Dodman (2001) in that noise phobia was significantly more represented in the group of dogs with separation anxiety compared to a control group. They go on to ascertain that almost half of the dogs in the separation anxiety group exhibited fear in response to noise compared to less than a third of the control dogs showing the same sort of fear.

In another study, Palestrini et al. (2010) reported that, based on owner's information, 60.8 percent of dogs with separation anxiety reacted to thunderstorms. It should be noted that this study had a very small sample size of 23 , so these results should be compared with other studies to confirm validity. However, there seems to be overwhelming evidence that both thunderstorm and noise phobia are correlated with separation anxiety. On an important note, Horwitz and Mills (2009) stressed that it is imperative that all the fears and anxieties are first identified and then treated in order for the separation anxiety to be successfully treated.

3.4.2 Aggression. Similarly, aggression is often reported alongside separation anxiety. McGreevy and Masters (2007) performed a univariate analysis to determine risk factors of separation anxiety and feed-related aggression by determining the significance of each of the predictor variables related to these such as new household members or dog excitability. It was discovered that food-related aggression was associated with a larger probability of a high separation-related distress $(P=0.007)$. Bamberger and Houpt's (2006) study using 1,644 dogs found that there were 58 incidences of dogs diagnosed with both general aggression and anxiety. The authors go on to explain that anxiety has the potential to lower the threshold of the dog for aggression. Dogs with aggression should be carefully observed for signs of separation anxiety and general anxieties, and owners should be questioned about the presence of this disorder. The authors go on to say that upward trends were detected in aggression and anxieties, including separation anxiety. Takeuchi et al. (2001) investigated the records of 448 dogs at the Cornell University Animal Behaviour Clinic over a five-year period to characterize three common behaviour problems in dogs: aggression towards owners, aggression towards strangers, and separation anxiety. The authors found that 32 dogs were diagnosed with both aggression towards people and separation anxiety. They chose to exclude these dogs for the purpose of their study, so they failed to discuss the significance of this finding. In another study by Storengen et al. (2014), the authors indicated that 32.6 percent of 215 dogs diagnosed with separation anxiety also had fear-related aggression and even more still had other aggression problems such as territorial aggression (2.3\%) and aggression in response to aversive painful interactions (4.2\%). They found that aggression was the second largest group of behaviours observed in the group of dogs with separation anxiety. This is a large proportion of these dogs, which suggests a correlation between separation anxiety and aggression.

\subsection{Environmental Implications of Separation Anxiety}

The environment that a dog is living in can have a significant impact on its mental health. This includes factors such as how the owner interacts with the dog, the housing situation, or the source of the dog. McGreevy and Masters (2007) explained that the most significant factor of ownerdog interaction affecting separation anxiety was gameplaying. Dogs which participated in game-playing with their owners had a higher probability of having a low separation related distress score $(P=0.023)$ compared to dogs that did not play. It should be noted that this is significantly altered if game-playing occurred within 30 minutes of owner's return home. Owners who played with their dogs within 30 minutes of returning home resulted in the dogs having a larger increase in probability of a high separation related distress score $(P=0.020)$. Similarly, if the dog was given a meal within 30 minutes of the owner's arrival, the dogs also had an increased probability of an elevated separation-related distress score $(P=0.050)$. They also explained that if a new human household member was introduced, the dog had a greater probability of having a higher score of separationrelated distress than those that had not $(P=0.012)$. If an owner underwent a job change, the dog also had an increased probability of an elevated separation-related distress score $(P=0.022)$. These last two points are logical because they would disrupt the dog's routine, which can cause anxiety. Flannigan and Dodman (2001) also found similar results. Interestingly, McGreevy and Masters (2007) found there was a correlation between a dog having a larger 
separation-related distress score and a higher number of adult females in the household $(P=0.014)$. Although the authors did not speculate why this result would occur, it could be related to the fact that female owners generally have higher pitched voices, smaller statures, and more nurturing demeanors. These could all contribute to a stronger bond because female owners are less intimidating. Flannigan and Dodman (2001) also looked at the environmental risk factors associated with separation anxiety. They found that more than three quarters of the dogs in their study with separation anxiety lived in households with multiple adults. However, dogs referred to the behaviour clinic were about two and a half times more likely to be diagnosed with separation anxiety if they came from a home with a single owner $(P<0.002)$. Although not explicitly stated, this could be because the dog has more opportunity to bond with this one person and form a hyperattachment. Additionally, there is a greater chance that a dog will be left home alone if there is only one member of the household.

Voith and Borchelt (1996) explained that many other factors can result in the development of separation anxiety, including the owner going on vacation and leaving the dog in a boarding kennel. Additionally, a major change in the household like a divorce, death of a family member, or a student leaving for college may also trigger this disorder. Lastly, even moving and leaving the dog in the new, strange house may result in separation-related behaviours when the dog was otherwise fine in the old house. Clearly, anything that disrupts the dog's daily routine or hierarchy within the home can trigger the development of separation anxiety.

\subsection{Housing Type}

The prevalence of canine separation anxiety also depends on what type of situation the dog is living in. For example, Takeuchi et al. (2001) found that there was a higher proportion of dogs with separation anxiety that were living in an apartment compared to a house. This could be a result of a lack of space in the apartment, or a greater tendency for a stronger attachment in smaller areas. Furthermore, dogs suffering from separation anxiety were more likely to be living in a more urban area, such as a city or town. However, this may be because neighbours are in closer proximity in cities and towns, so vocalization of the dogs will be more frequently reported. It is imperative that more research be performed before this result is determined to be significant. Similar results were found by Palestrini et al. (2010) in that 60 percent of the dogs in their study with separation anxiety lived in an apartment, compared to only about a third of the dogs living in a house.

\subsection{Source of Dog}

The prevalence of separation anxiety can vary depending on where a dog originates from. This can range from pet stores and breeders, to secondary sources such as shelters or family friends. Interestingly, McGreevy and
Masters (2007) found that the acquisition of dogs was the most significant factor when it came to separation anxiety. They found that there was a higher probability of a dog being acquired from friends or family having a low separationrelated distress score compared to dogs that came from a pet store. This could be because the dog has been introduced to the owner before they acquire the dog, which would result in a smoother transition into their new home.

4.2.1 Shelters. Shelters and rescue operations are a common source of dogs in society today. Despite the staff's best efforts, these places can be stressful for the dogs. These dogs have been abandoned by their owners or were lost, and they are surrounded by unfamiliar dogs, people, and environments. This environmental stress can result in the development of many behavioural issues, including separation anxiety (Sergurson et al. 2005). Voith and Borchelt (1996) theorized that perhaps the period of the dog feeling abandoned results in it forming a very strong attachment to its new owner because opportunity has presented itself. Sherman and Mills (2008) explained that it is common for shelter dogs to exhibit separation anxiety because they may have had an experience in their past that predisposes them to the disorder, or they may be the offspring of dogs that suffered from it. They emphasized that it is also common for dogs with separation anxiety to be repeatedly returned to shelters and readopted because of the difficulty in managing such dogs. Flannigan and Dodman (2001) found that dogs which were abandoned to a shelter and then adopted by new owners were more likely to develop separation related distress compared to dogs from a breeder, friend, or pet store $(P=0.017)$. It was also found that dogs which spent excessive amounts of time in pet stores before they are purchased had a similar likeliness to develop separation anxiety. Additionally, they reported that 41.1 percent $(n=79)$ of the 192 dogs with separation anxiety in the study came from shelter-type sources, compared to only 29.6 percent $(58 / 196)$ of the control dogs from this source.

In addition to the possibility that dogs are more likely to have separation anxiety if they come from a shelter, Takeuchi et al. (2000) found that, in their follow-up telephone interview after discharge instructions were given to the owners, these dogs were significantly less likely to improve (43\%) compared to dogs from other sources $(77 \%)$. It is clear shelter staff must give utmost focus to treating separation anxiety to ease the transition of the dog into its new home (Takeuchi et al. 2000).

In contrast, Palestrini et al.'s 2010 study found that only 25 percent of dogs with separation anxiety were adopted from a shelter, while 31.2 percent were acquired from another person without reporting if they knew the person or not. They specified that the source of the dog had no significant effect on separation-related behaviours. Comparably, Bradshaw et al. (2002) reported that their data 
did not support the common assumption that dogs who were rescued or rehomed had a greater likelihood of developing separation anxiety compared to dogs from breeders. A notable factor in regard to the effects of shelters on separation anxiety is that none of the above studies distinguished the shelter conditions such as the training or number of staff, number of dogs, housing conditions, or exercise protocols. These and multiple other factors could affect a dog's wellbeing and thus alter the likelihood of a separation anxiety diagnosis.

\subsection{Separation Anxiety Mitigations}

Canine separation anxiety can clearly result in many detrimental effects and must be mediated properly. Overall et al. (2001) concluded that early intervention is of the utmost importance, and that any time a dog displays the symptoms, a veterinarian should determine if a diagnosis for separation anxiety is valid. They also stressed that dogs who had previously suffered separation anxiety that had been resolved were at a much higher risk of developing severe symptoms if it reoccurred. As Voith and Borchelt (1996) explained, behavioural disorders can result due to many different causes. Because of this, they stress that a differential diagnosis must occur to determine the appropriate treatment for a certain canine. Horwitz and Mills (2009) described a differential diagnosis as being based on key findings in the behavioural history while excluding other behavioural and medical problems. Successful treatment for separation anxiety includes a combination of environmental, behavioural, and pharmacological approaches (Sherman 2008; Horwitz and Mills 2009). Treatment of this disorder has the potential to improve or eliminate symptoms while repairing and bettering the bond between dog and owner (Horwitz 2000).

\subsection{Training}

Training both the dogs and the owners is imperative to mediating separation anxiety. Bradshaw et al. (2002) stresses that separation-related anxiety is mainly due to the relationship between the dog and owner. Despite this significance, they stressed that only a small portion of owners actually reach out for help if their dogs are suffering from this disorder. They also stated that it is important to provide the dog with a wide variety of experiences between around five to ten months of age, such as varying times of the owner's absence or interactions with people. This will ideally result in more tolerable behaviour in the future. They attributed this to the fact that dogs have descended from wolves, which are pack animals. Wolves constantly interact with their pack, which can be linked to a dog's necessity for human interaction. If there is a sudden drop in levels of interaction with people, this may predispose the dog to separation anxiety in the future. In addition to this, Sherman (2008) stressed the importance of owners ignoring clingy and attention-seeking behaviour. This is important to prevent the dogs from forming hyperattachment to their owners. The hope is that the dog will eventually learn to lie down while the owner is moving throughout the house or exhibiting departure cues.

Although efficient for some other behavioural issues, it is stressed by Voith and Borchelt (1996) that punishment is not an efficient way to treat separation anxiety, and that it actually has the potential to increase it. This is because, when using punishment for training, it must be administered every time the dog is exhibiting the unwanted behaviour in that exact moment. This is evidently not feasible since dogs with this disorder only exhibit the symptoms while the owner is away. If punishment is inappropriately given when the owner returns, the dog may associate their return with the punishment and develop an increased anxiety response. Both Horwitz and Mills (2009) and Sherman (2008) also agree that owners should be encouraged to cease punishment for misbehaviors that may occur while they are away. In place of punishment, many training techniques can be utilized and tailored for each individual dog, with two common ones being counter-conditioning and desensitization.

5.1.1 Counter-conditioning. In conjunction with the aforementioned techniques, Voith and Borchelt (1996) recommended to counter-condition the dog's anxiety while the owner is preparing to depart. This involved making the dog sit and stay or introducing a food reward or praise so that this is associated with the departure. Horwitz and Mills (2009) explained that this technique is most successful when the owner makes the dog sit and stay while gradually increasing the time and distance that they are apart before rewarding them with a treat.

5.1.2 Desensitization. Voith and Borchelt (1996) explained that the underlying basis of treating any anxiety should be the gradual introduction to the fear without eliciting a response. When it comes to canine separation anxiety, they recommend gradually allowing the dog to get used to being left alone while slowly increasing the length of time they are alone. The time of the owner's departure should only be increased if the dog shows no predeparture anxiety and does not display overexcited behaviour when the owner has returned. If successful, this training will result in the gradual increase in the time spent alone without eliciting an anxiety response. Similarly, Appleby and Pluijmakers (2004) explained that each animal's behavioural treatment should be tailored to them specifically, and that any treatment should be administered in phases to avoid additional anxiety. In a small study by Butler et al. (2010), the authors concluded that utilizing desensitization resulted in decreasing both the severity and frequency of separation anxiety symptoms. 
5.1.3 Combining Training Techniques. It is commonly recommended that multiple training techniques be used to treat dogs with separation anxiety. Through local advertisements, Blackwell et al. (2006) gathered 55 dogs that had displayed symptoms of separation anxiety for at least a month. They were separated into two treatment groups and one control group. The owners of the dogs in both treatment groups (defined below) were given a comprehensive behaviour modification program while they participated in a standardized consultation. The program consisted of three phases; the first phase was designed to decrease the dog's dependency on the owner by ignoring its attention-seeking actions. The next phase of the program consisted of desensitizing the dog to the fact that it is left alone by slowly increasing the length of departure and by decreasing the predictability of the departure. The last phase involved providing the dog with something to preoccupy itself while it is alone, such as a special toy or something that smells like the owner. The program emphasized not punishing the dogs for misbehavior when the owner returns. The two treatment groups differed only in the order that the components of the program were presented. After 12 weeks of the program, 56 percent of the owners reported that they saw a significant improvement in their dogs, with no significant difference between the treatment groups. It should be noted that this study used a relatively small sample size. Ultimately though, the results showed strong evidence that such treatment programs can be effective for treating separation anxiety, with only two owners reporting a slight worsening on their dog's condition. Later, Blackwell et al. (2016) found similar results. New owners of shelter dogs were given written instructions on preventing separation anxiety in their dogs. The authors found that these dogs were less likely to develop this condition compared to control dogs.

Takeuchi et al.'s 2000 study provided individualized discharge instructions to 110 owners of dogs with separation anxiety. The instructions included ceasing punishment, increasing exercise, desensitizing the dog to departures by leaving for short periods of time, and providing a toy at departure. After a minimum of six months, 52 owners that still had their dogs were contacted on the phone and questioned as to whether or not the treatment was successful, as well as how strictly they followed the instructions. Sadly, it was determined that six of the dogs had been euthanized and four had been given away. Of the owners who still had their dogs, three reported that their dogs were worse, seven thought their dogs had not changed, twenty-four believed their dog's condition had improved, and eight said their dog's condition had been cured. Crucially, this study also investigated whether the owners complied with certain instructions, and what the optimal number of instructions was. They discovered that significantly fewer dogs improved or were cured if their owners were given more than five instructions compared to those given five or fewer. Interestingly, the outcome of the treatment was not different regardless of which instructions the owners had followed. Owners were more likely to follow no punishment $(79 \%)$, increased exercise $(78 \%)$ and providing a toy $(69 \%)$, while only 43 percent of owners complied with desensitization. This study went to even greater lengths to see how effective treatments were by asking the owners their opinions of the treatments. 74 percent of the owners believed that downplaying their departure was effective, and 46 percent of owners thought that sit-and-stay relaxation, or counter-conditioning, was also effective.

\subsection{Medication}

Voith and Borchelt (1996) found that anti-anxiety medications can be effective when leaving dogs suffering from separation anxiety for prolonged periods of time. They explained that these drugs may not be effective in extreme cases of the disorder, but in mild cases, the dose can slowly be reduced until the dog becomes used to dealing with the separation on its own. They stressed that a good anti-anxiety medication does not sedate the dog, and that sedation could potentially reduce the dog's learning ability. These drugs instead temporarily slow down the nervous system through various mechanisms (noted below). It is important to note that no behavioural medication is equally effective for all dogs (Sherman 2008). Medications have been proven to be effective in many studies, but it is unclear if they inhibit certain behaviours or if it is an improvement in the affective, or emotional, state (Karagiannis et al. 2015). Sherman and Mills (2008) explained that there are two psychotropic medications approved by the United States Food and Drug Administration for use in treating canine separation anxiety. These two drugs are clomipramine (Clomicalm) and fluoxetine (Reconcile). Clomipramine is a commonly prescribed tricyclic compound that is a potent serotonin reuptake inhibitor, and fluoxetine is a more recently approved selective serotonin reuptake inhibitor.

5.2.1 Clomipramine. Many studies have looked at the efficacy of clomipramine in treating canine separation anxiety. Clomipramine is a potent and selective reuptake inhibitor of neuronal serotonin and also inhibits neuronal noradrenaline reuptake, which results in the mediation of anxiety symptoms (King et al. 2000). In a recent study, Cannas et al. (2014) investigated the effects of this medication on 23 dogs that had presented separation anxiety symptoms at two behavioural clinics. This study utilized a video camera to monitor the dog's behaviour while it was home alone. Clomipramine significantly reduced whining, pacing, and scratching in only one week while significantly increasing relaxation behaviours such as resting and sleeping. After two weeks, the dose was increased, and barking decreased. Overall, they found that clomipramine 
efficiently reduced the symptoms of canine separation anxiety, which also improved welfare. Although this study used a very small sample size, the use of video cameras ensured accurate, nonbiased reports of the efficacy of this drug. Similar effects were seen in other studies as well (Gaultier et al. 2005).

In an earlier study, King et al. (2000) recruited 95 dogs with separation anxiety from veterinarians and advertisements. The dogs were randomly allocated into three groups, including placebo, "low-dose," and "standard dose" of clomipramine. This was a randomized, double-blind clinical trial. All dogs also received behavioural therapy, which may alter the validity of the results, but makes it more representative of an authentic treatment program. The results were derived from questionnaires that the owners completed. When comparing the results to the placebo group, the low dose group showed no significant difference in behaviour. The standard-dose group, however, improved more than three times quicker than the placebo group, which was evident in the reduction of destruction, defecation, and urination as well as the owner's overall assessment of the dog's behaviour. No significant difference was seen when comparing the standard-dose and placebo groups in regard to the reduction of vocalization. It should be noted that, in a small number of dogs, minor vomiting was observed as a side effect of clomipramine supplementation. In order to look at the long-term effects of this medication, King et al. (2004) contacted owners of 78 dogs from the original study. No cases of adverse effects were detected in dogs on clomipramine long term for more than 13 months. The owners found significant improvements in the disorder due to the standard-dose. The low-dose was still not significantly different compared to the placebo. Ultimately, no long-term adverse effects were reported due to this medication.

5.2.2 Fluoxetine. Fluoxetine is another medication that has been proven to be effective in various studies. Similar to clomipramine, fluoxetine treats anxiety by enhancing serotonin levels at presynaptic axon terminals by acting as a selective serotonin reuptake inhibitor (Simpson et al. 2007). Simpson et al. (2007) performed a double-blind, placebocontrolled study based on this drug. This study incorporated 242 dogs with separation anxiety randomly into a treatment or placebo group, with both groups participating in behavioural treatment as well. The treatment group received $1-2 \mathrm{mg} / \mathrm{kg} /$ day of fluoxetine. Results were based on owner feedback of the behavioural changes in their dogs. There was a 42 percent improvement of dogs in the fluoxetine treatment group, compared to only 17 percent of the placebo group by week one $(P=0.003)$. At week eight, 72 percent of the dogs in the treatment group had improved compared to only 50 percent of the placebo group. The improvement observed in the placebo group was most likely attributed to the success of behavioural modification.
Overall, this medication has been shown to be effective in a large sample of dogs, with the only adverse effects reported being transient lethargy and a reduction in appetite.

In a more recent study, Karagiannis et al. (2015) looked at the effects of fluoxetine tablets on five dogs with separation anxiety while they concurrently participated in behavioural treatment for two months. Seven dogs without separation-related problems were used as a control. The owners responded to questions regarding their progress and a spatial cognitive bias test was performed. These tests measure the affective state of animals. This involves training the dogs to discriminate between a bowl with food and a bowl without food at two different locations. A dog with a more "optimistic" bias is seen as running faster to unrewarded areas between learned locations whereas a "pessimistic" dog would be seen as running slower. As a whole, this study found that treating a negative affective state and the associated behaviours of separation anxiety with fluoxetine was successful, with a significant shift in cognitive bias. They concluded that not only did the dog's behaviour improve, but also their welfare. It should be noted that both of these studies lacked the detail of looking at long-term effects of fluoxetine.

\subsection{Impacts of Separation Anxiety}

Canine separation anxiety has the potential to irreparably deteriorate the bond that an owner and dog share (Sherman and Mills 2008). Despite canine separation anxiety seemingly not posing a major health risk to the dog, frustrated owners may choose to surrender the animal to a shelter or decide to have it euthanized (Simpson et al. 2007). This could be a result of emotional frustration or monetary stresses due to damage of their home (Sherman and Mills 2008). Although these are unfortunate outcomes, they are all too real possibilities if proper treatment is not available. In fact, Salman et al. (2000) investigated the relinquishment records of 12 shelters and found that the most often recorded reason was behavioural issues. Although not explicitly mentioned, many of these issues listed are likely symptoms of canine separation anxiety, including excessive vocalization, the animal needing too much attention, destructiveness, and fear. This study used a very large sample size $(1,984)$ and accounted for a good geographical distribution via the 12 different shelters.

Takeuchi et al. (2000) stressed that behavioural advice for treatment of canine separation anxiety should be made available to veterinarians, in order to attempt to decrease the number of dogs euthanized unnecessarily as a result of this disorder. As mentioned previously, six of the 52 dogs used in the study were euthanized and four were given away. This could have been avoided with the development of an effective treatment regimen. In another study by Sergurson et al. (2005), 54 dogs being relinquished to a shelter had a higher chance of having separation-related anxiety 
compared to 784 control dogs owned by veterinary clients. They attested this to the fact that this disorder fell in a category that would affect how difficult it is to keep the dog. They also postulate that perhaps the reason that some studies (Takeuchi et al. 2000; Flannigan and Dodman 2001) have found a higher prevalence of separation anxiety in dogs adopted from shelters is because it is more common for these dogs to be relinquished in the first place.

Bamberger and Houpt (2006) reported that there is an upward trend in canine separation anxiety. Because of this, it is imperative that veterinarians ensure that their knowledge of the disorder is current, so that they are best equipped to diagnose and prescribe appropriate treatment and medication for the dog, in order to prevent unnecessary euthanasia or relinquishment to shelters. In other words, proper treatment of separation anxiety will not only result in an improved relationship between the dog and its owner, but also result in fewer animals being surrendered to shelters (Thielke and Udell 2017)

\subsection{Conclusion}

Canine separation anxiety is a widespread, multifactorial disorder that is influenced by numerous factors. Some of these factors have many studies supporting their effect on this disorder, while others continue to be heavily debated. The most common clinical signs of canine separation anxiety include excessive vocalization, destruction throughout the house, inappropriate elimination, and excessive motor activity. The scope of symptoms is vast, and crosses over with other behavioural disorders, making an accurate diagnosis difficult. Some factors that are thought to affect separation anxiety in dogs are the degree of attachment to the owner, gender, breed, and existing behavioural issues such as noise and thunderstorm phobia or aggression. In addition to these, environmental factors can predispose a dog to separation anxiety, such as the housing situation and the source of the dog.

Fortunately, if a diagnosis of canine separation anxiety is made, there are many mediations that are proven to be effective in treating this disorder. These include training techniques such as desensitization and counterconditioning. It was found, however, that combining many behavioural modification techniques was the most effective. In conjunction with this, medications have been found to be very effective without causing long-term adverse effects. Two currently approved and proven medications include fluoxetine and clomipramine.

Despite the existence of many effective mitigations, many owners experience emotional and financial strain in managing the various symptoms that this disorder presents. This results in the all-too-common outcome of either relinquishing the dog to a shelter or euthanizing it. It is because of this, and the welfare of the animal, that canine separation anxiety should be diagnosed as early as possible and treated efficiently.

As it remains controversial which factors affect separation anxiety, additional studies will be required in which determining these factors should be the main objective. This is important because there were few studies with the specific factors being the focus, instead of just a type of measurement. Also, a more accurate diagnostic metric needs to be developed so that veterinarians may detect separation anxiety in a quicker manner. More studies should also investigate whether or not the predictability of the owner's departure cues is beneficial or harmful to the dog. Additionally, since shelters seem to be the source of a higher majority of dogs with separation anxiety, and dogs with this disorder are relinquished there, shelter staff should be informed of how to detect and treat the condition to improve the adoptability and long-term welfare of the dogs. Another opportunity for future studies is focusing on the underlying physiology of why canine separation anxiety occurs. Ultimately, canine separation anxiety is a disorder that is becoming increasingly common and must be efficiently diagnosed and treated to better the welfare of both the dog and its owner.

\section{Acknowledgements}

I would like to offer my sincerest gratitude to Professor Karen Schwean -Lardner for her immeasurable guidance and support through this process. This paper would not be successful had it not been for her sharing of knowledge and willingness to help whenever needed. 


\section{References}

Amat, M., Camps, T., Brech, S. L. and Manteca, X. 2014 Separation anxiety in dogs: the implications of predictability and contextual fear for behavioural treatment. Anim. Welfare. 23: 263-266.

Appleby, D. and Pluijmakers, J. 2004. Separation anxiety in dogs: The function of homeostasis in its development and treatment. Clin. Tech. Small An. P. 19: 205-215.

Bamberger, M. and Houpt, K. 2006. Signalment factors, comorbidity, and trends in behavior diagnoses in dogs: 1,644 cases (1991-2001). J. Am. Vet. Med. Assoc. 229: 1591-1601.

Blackwell, E. J, Casey, R. A. and Bradshaw, J. W. S. 2006. Controlled trial of behavioural therapy for separation related disorders in dogs. Vet. Rec. 158: 551-554.

Blackwell, E. J., Casey, R. A. and Bradshaw, J. W. S. 2016. Efficacy of written behavioral advice for separationrelated behaviour problems in dogs newly adopted from a rehoming center. J. Vet. Behav. 12: 13-19.

Bradshaw, J. W. S., McPherson, J. A., Casey, R. A. and Larter, I. S. 2002. Aetiology of separation related behaviour in domestic dogs. Vet. Rec. 151: 43-46.

Butler, R., Sargisson, R. J. and Elliffe, D. 2010. The efficacy of systematic desensitization for treating the separation-related problem behaviour of domestic dogs. Appl. Anim. Behav. Sci. 129: 136-145.

Cannas, S., Frank, D., Minero, M., Aspesi, A., Benedetti, R. and Palestrini, C. 2014. Video analysis of dogs suffering from anxiety when left home alone and treated with clomipramine. J. Vet. Behav. Clin. Appl. Res. 9: 50-57.

Flannigan, G. and Dodman, N. H. 2001. Risk factors and behaviors associated with separation anxiety in dogs. J. Am. Vet. Med. Assoc. 219: 460-466.

Gaultier, E., Bonnafous, L., Bougrat, L., Lafont, C. and Pageat, P. 2005. Comparison of the efficacy of a synthetic dog-appeasing pheromone with clomipramine for the treatment of separation-related disorders in dogs. Vet. Rec. 156: 533-538.

Horwitz, D. F. 2000. Diagnosis and treatment of canine separation anxiety and the use of clomipramine hydrochloride (clomicalm). J. Am. Anim. Hosp. Assoc. 36: 107-109.
Horwitz, D. F. and Mills, D. S. 2009. BSAVA manual of canine and feline behavioural Medicine. 2nd Ed. British Small Animal Veterinary Association, Gloucester, UK. 146-158 pp.

Karagiannis, C. I., Burman, O. H. P. and Mills, D. S. 2015. Dogs with separation related problems show a "less pessimistic" cognitive bias during treatment with fluoxetine (Reconcile ${ }^{\mathrm{TM}}$ ) and a behaviour modification plan. Bmc. Vet. Res. 11:1-10.

King, J. N., Overall, K. L., Appleby, D., Simpson, B. S., Beata, C., Chaurand, C. J. P., Heath, S. E., Ross, C., Weiss, A. B., Muller, G., Bataille, B. G., Paris, T., Pageat, P., Brovedani, F., Garden, C. and Petit, S. 2004. Results of a follow-up investigation to a clinical trial testing the efficacy of clomipramine in ${ }^{-}$the treatment of separation anxiety in dogs. Appl. Anim. Behav. Sci. 89: 233-242.

King, J. N., Simpson, B. S., Overall, K. L., Appleby, D., Pageat, P., Ross, C., Chaurand, J. P., Heath, S., Beata, C., Weiss, A. B., Muller, G., Paris, T., Bataille, B. G., Parker, J., Petit, S. and Wren, J. 2000. Treatment of separation anxiety in dogs with clomipramine: results from a prospective, randomized, double-blind, placebo-controlled, parallel-group, multicenter clinical trial. Appl. Anim. Behav. Sci. 67: 255-275.

Martinez, A. G., Pernas, G. S., Casalta, F. J. D., Rey, M. L. S. and Palomino, L. F. D. L, 2011. Risk factors associated with behavioral problems in dogs. J. Vet. Behav. 6: 225-231.

McGreevy, P. D. and Masters, A. M. 2007. Risk factors for separation-related distress and feed-related aggression in dogs: Additional findings from a survey of Australian dog owners. Appl. Anim. Behav. Sci. 109: 320-328.

Overall, K. L., Dunham, A. E. and Frank, D. 2001. Frequency of nonspecific clinical signs in dogs with separation anxiety, thunderstorm phobia, and noise phobia, alone or in combination. J. Am. Vet. Med. Assoc. 219: 467-473.

Palestrini, C., Minero, M., Cannas, S., Rossi, E. and Frank, D. 2010. Video analysis of dogs with separation-related behaviors. Appl. Anim. Behav. Sci. 124:61-67. 
Parthasarathy, V. and Crowell-Davis, S. L. 2006. Relationship between attachment to owners and separation anxiety in pet dogs (Canis lupus familiaris). J. Vet. Behav. Clin. Appl. Res. 1: 109-120.

Salman, M. D, Hutchison, J., Ruch-Gallie, R., Kogan, L., New Jr, J. C., Kass, P. H. and Scarlet J. M. 2000. Behavioral Reasons for Relinquishment of Dogs and Cats to 12 Shelters. J. Appl. Anim. Welf. Sci. 3: 93-106.

Segurson, S. A., Serpell, J. A. and Hart, B. L. 2005. Evaluation of a behavioral assessment questionnaire for use in the characterization of behavioral problems of dogs relinquished to animal shelters. J. Am. Vet. Med. Assoc. 227: 1755-1761.

Sherman, B. L. 2008. Separation anxiety in dogs. Compend. Contin. Educ. Vet. 30: 27-42.

Sherman, B. L. and Mills, D. S. 2008. Canine anxieties and phobias: an update on separation anxiety and noise aversions. Vet. Clin. Small Anim. 38: 1081-1106.

Simpson, B. S. 2000. Canine separation anxiety. Comp. Cont. Educ. Pract. 22: 328-338.

Simpson, B. S., Landsberg, G. M., Reisner, I. R., Ciribassi, J. J., Horwitz, D., Houpt, K. A., Kroll, T. L., Luescher, A., Moffat, K. S., Douglass, G., Robertson-Plouch, C., Veenhuizen, M. F., Zimmerman, A. and Clark, T. P. 2007. Effects of reconcile (fluoxetine) chewable tablets plus behavior management for Canine separation anxiety. Vet. Ther. 8: 18-31.

Storengen, L. M., Boge, S. C. K., Strøm, S. J., Løberg, G. and Lingaas, F. 2014. A descriptive study of 215 dogs diagnosed with separation anxiety. Appl. Anim. Behav. Sci. 159: 82-89.

Takeuchi, Y., Houpt, K. A. and Scarlett, J. 2000. Evaluation of treatments for separation anxiety in dogs. J. Am. Vet. Med. Assoc. 217: 342-345.

Takeuchi, Y., Ogata, N., Houpt, K A. and Scarlett, J. 2001. Differences in background and outcome of three behavior problems of dogs. Appl. Anim. Behav. Sci. 70:297-308.

Thielke, L. E. and Udell, A. R. 2017. The role of oxytocin in relationships between dogs and humans and potential applications for treatment of separation anxiety in dogs. Biol. Rev. 92: 378-388.
Voith V. L. and Borchelt P. L. 1996. Readings in companion animal behavior. Veterinary Learning Systems, Trenton, NJ. 124-139 pp. 
